\title{
Anal squamous cell carcinoma in KwaZulu-Natal Province, South Africa, with special reference to the influence of HIV infection on clinical presentation and management outcome
}

\author{
N P Zuma, ${ }^{1}$ MB ChB, FC Rad Onc (SA); S Ngidi, ${ }^{1}$ MB ChB, FC Rad Onc (SA), MMed; \\ T E Madiba, ${ }^{2} \mathrm{MB}$ ChB, MMed, LLM, PhD, FCS (SA), FASCRS \\ ${ }^{1}$ Department of Radiation Oncology, School of Clinical Medicine, College of Health Sciences, University of KwaZulu-Natal, Durban, South Africa \\ ${ }^{2}$ Gastrointestinal Cancer Research Centre, School of Clinical Medicine, College of Health Sciences, University of KwaZulu-Natal, Durban, South Africa
}

Corresponding author: N P Zuma (drnpzuma@gmail.com)

Background. Anal carcinoma is rare. Clinicopathological features influencing outcome have not been determined in HIV-negative and HIV-positive patients in South Africa.

Objectives. To compare presentation and treatment tolerance among HIV-positive and negative patients.

Methods. This study was a retrospective analysis of prospectively collected data on patients with squamous cell carcinoma of the anus. Patients with known HIV status were extracted from the anal cancer database and analysed. Data analysed included demographics, clinical features, stage, pathology and treatment outcome.

Results. There were 268 patients with anal squamous cell carcinoma ( $n=135$ HIV-positive and $n=33$ negative). The median age was 39 years and 53 years for HIV-positive and negative patients, respectively, the male/female ratio was 1:2.7 and 1:1.8 for the two groups, and the ratio of anal margin to canal distribution was 1.3:1 and 1:1. Disease stage was similar, with minor differences. The resection rate was $17 \%$ in HIV-positive patients and $9 \%$ in those who were HIV-negative. Half the patients in both groups were eligible for definitive therapy, and side-effects of oncotherapy occurred with similar frequency in both groups. The recurrence rate was $7 \%$ in both groups and the disease-free interval was similar. Overall survival was longer for HIV-negative patients $(p=0.0240)$.

Conclusions. The prevalence of anal squamous cell carcinoma is much higher in individuals with HIV infection than in those who are HIVnegative. HIV-positive patients present at a younger age and with locally advanced disease that responds less well to standard treatment, and their survival is poorer.

S Afr Med J 2020;110(3):243-248. https://doi.org/10.7196/SAMJ.2020.v110i3.14290

Anal cancer is rare, comprising $\sim 2.7 \%$ of all malignancies of the digestive system. ${ }^{[1]}$ The Surveillance, Epidemiology, and End Results (SEER) Program of population-based tumour registries in the USA estimated that there were 8580 new cases in 2018. ${ }^{[2]}$ The incidence of anal cancer has increased over the past three decades, both in the USA and elsewhere. ${ }^{[3,4]}$ The clinicopathological features of patients with anal squamous cell carcinoma and HIV infection remain largely unknown.

Squamous cell carcinoma is the most common anal cancer, followed by adenocarcinoma, and the least common varieties are melanoma, lymphoma and neuroendocrine tumours. ${ }^{[5]}$ Anal cancer can occur in the anal canal or the anal margin, whereas adenocarcinoma occurs solely in the anal canal.

Risk factors associated with an increased incidence of anal squamous cell carcinoma include female gender, human papillomavirus (HPV) infection, especially subtypes 16 and 18 , immunosuppression, anoreceptive intercourse, a high lifetime number of sexual partners, sexually transmitted diseases, genital warts, smoking and HIV infection. ${ }^{[3,4,6,7]}$

Immunosuppression has in turn been shown to accelerate the progression of HPV-associated anal precursor lesions such as anal intraepithelial neoplasia (AIN). ${ }^{[8]}$ The incidence of anal cancer is markedly elevated among people with HIV infection, especially in men who have sex with men (MSM), ${ }^{[9-1]}$ and $>40 \%$ of patients with HIV infection develop a cancer of some sort during their life- time ${ }^{[12]}$ However, although anal cancer is one of the HIV-associated malignancies (HIVAM), it has not been identified as AIDS defining. ${ }^{[13]}$

Studies on carcinogenesis have highlighted the role of immunosuppression in the accelerated progression of HPV-associated anal precursor lesions. ${ }^{[4]}$ Whereas the introduction of antiretroviral therapy (ART) has led to improvement of the immune system of infected individuals and substantially increases their life expectancy, ${ }^{[14]}$ it has not been shown to reduce the prevalence of HPV infection, which is the causative agent in $>90 \%$ of invasive anal carcinomas. ${ }^{[15]}$ It therefore appears that the extended survival of HIV-positive individuals on ART coupled with the inability of ART to restore adequate host immune system function to combat HPV infection and progression to malignancy may explain why co-infection with HIV and HPV increases HIVAM. ${ }^{[13,14]}$

There are limited international studies addressing HIV and anal cancer. Despite sub-Saharan Africa being an epicentre of HIV infection, there are no data on HIV infection and anal cancer from Africa. Furthermore, there is little South African (SA) literature reporting on anal cancer, and none addressing outcomes in HIVpositive and HIV-negative patients. International studies can be criticised on the grounds of the small numbers of HIV-infected patients with anal cancer reported and the fact that their data cannot be generalised to the African context, including SA, hence the need to address this question from a developing country like SA. 


\section{Objectives}

We hypothesised that the clinical presentation of anal cancer in HIV-positive patients differs from that in HIV-negative patients. In an attempt to address the dearth of knowledge in this area, we undertook a retrospective cohort study of patients with squamous cell carcinoma of the anus to determine the clinicopathological factors that influence outcome in HIV-infected and non-infected patients. The study analysed an ongoing anal cancer database in KwaZuluNatal Province, SA. Our objective was to determine the demographic profile of patients with anal cancer, with special attention to HIV infection. This is the first study with a large study population to address the clinicopathological spectrum of anal cancer in SA.

\section{Methods \\ Study setting}

The study was undertaken in the colorectal unit at Inkosi Albert Luthuli Central Hospital (IALCH), a tertiary referral hospital in Durban, SA.

\section{Study population}

The study population was previously untreated patients newly diagnosed with invasive anal squamous cell carcinoma and managed at IALCH. All patients with invasive squamous cell carcinoma were included in the study, and those with anal malignancy other than squamous cell carcinoma were excluded. Patients whose HIV status was known were divided into HIV-negative and positive groups and analysed separately. Patients with unknown HIV status were excluded from the subsequent subanalysis.

\section{Study design}

This was a retrospective cohort study of data collected from the ongoing anal cancer database archived in the Gastrointestinal Cancer Research Centre of the University of KwaZulu-Natal. Patients with anal squamous cell carcinoma were extracted from the database, which spanned a period of 18 years from 2000 to 2017. The dataset analysed included demographic data such as age, gender, tumour stage, HIV status, CD4+ count, treatment received and treatment outcome. The TNM Classification of Malignant Tumours (8th edition) and Union for International Cancer Control (UICC) staging were used during data capture. For the purposes of this article, the UICC staging was used. The following protocol is followed in our unit for the management of patients with anal squamous cell carcinoma. Patients who present with lesions suspicious of anal cancer are initially evaluated in the regional hospitals across the province. Once the diagnosis is confirmed and appropriate staging investigations have been undertaken, they are then referred to the Oncology Unit at IALCH, where they are evaluated in a multidisciplinary clinic. Further management is then discussed at a multidisciplinary board meeting where a treatment plan is decided on based on clinical features, staging and patient performance status.

\section{Therapeutic modalities}

Patients with stage I disease were treated with primary resection, which involved either wide local excision or abdominoperineal resection. Patients requiring definitive therapy received chemoradiation. The chemotherapy arm used either a single-agent regimen such as capecitabine, cisplatin or carboplatin or combination chemotherapy comprising cisplatin plus mitomycin, cisplatin plus 5 -fluorouracil, or mitomycin plus capecitabine. The radiation technique varied from three-dimensional conformal radiation to volumetric arc therapy with a combination of photons and electron boost to the primary and inguinal nodes. The average radiation dose was 45 Gy to the primary tumour and pelvic nodes and a 14.4 Gy electron boost to the primary and inguinal nodes where deemed necessary. All patients were initially reviewed 6 weeks after completion of treatment and again 6 weeks later. The response to treatment was assessed clinically. If the size of the primary tumour had decreased by $50 \%$, the clinical response was assessed as partial; complete disappearance of disease was considered complete clinical response. Persistent non-progressive anal lesions were biopsied to confirm the presence of malignancy or to demonstrate complete pathological response. Palliative radiation was delivered to the primary lesion at various doses in patients with advanced disease such as stage IV disease or those with poor performance status.

\section{Data management and statistical analysis}

Data were stored on an Excel spreadsheet, version 2016 (Microsoft, USA) and analysed using the Statistical Package for the Social Sciences (SPSS) (IBM, USA). Dates of death were identified where possible via the Department of Home Affairs. Quantitative data were expressed as medians and interquartile ranges (IQRs) and comparison was made using non-parametric tests. Actuarial survival was analysed using the Kaplan-Meier method. The level of significance was set at $p<0.05$.

\section{Ethical considerations}

Ethical approval was obtained from the Biomedical Research Ethics Committee of the University of KwaZulu-Natal (ref. no. R057/04).

\section{Results}

Fig. 1 illustrates the inclusion and exclusion criteria that led to a total of 268 patients with squamous cell carcinoma being eligible for initial analysis. One hundred and sixty-eight patients agreed to voluntary counselling and testing for HIV infection, of whom 135 were HIVpositive and 33 were HIV-negative (Table 1). One hundred patients ( $n=35$ males) were not tested for HIV, either because they refused or were not asked to undergo the test. HIV-positive patients were a decade younger than their HIV-negative counterparts $(p<0.0001)$. Female patients predominated in the cohort as a whole, and the proportion of female patients was relatively higher among HIVpositive patients compared with HIV-negative patients. Anal margin disease predominated in the whole group, and this predominance was more pronounced among HIV-positive patients than among HIV-negative patients. The median duration of symptoms for both the HIV-positive and negative groups was 12 (IQR 2 - 24) months. Fig. 2 demonstrates the annual accrual of patients with squamous cell carcinoma. There was a trend towards increasing diagnosis of this cancer over the years.

Staging is shown in Fig. 3. The majority of patients had stage III disease, with a higher proportion of HIV-positive patients than HIVnegative patients. There was a slight increase in the proportion of HIV-negative patients in stages II and IV. The trends in staging did not reach statistical significance.

Primary management is shown in Table 2. The proportion of patients receiving definitive therapy was similar in the two cohorts. A higher proportion of HIV-negative than HIV-positive patients received palliative therapy ( $36 \%$ v. $24 \%$ ), and this difference in treatment objectives between the two groups did not reach statistical significance $(p=0.181)$. The proportion of patients who did not receive any treatment at all was higher among HIV-positive patients than among those who were HIV-negative ( $24 \%$ v. $12 \%)$. Reasons for patients receiving no treatment included failure to return, prior resection and poor performance status. Complete clinical response 
was seen in 10/99 HIV-positive patients (10\%) compared with 5/30 HIV-negative patients $(17 \%)$, and complete pathological response in 6/99 HIV-positive patients (6\%) compared with 2/30 HIV-negative patients (7\%). Two of the HIV-positive patients died while receiving radiation therapy.

Surgical management is set out in Table 3. The resection rate among HIV-positive patients was $13 \%$, compared with $9 \%$ among HIV-negative patients. There was no difference in terms of up-front v. salvage resection. Two HIV-positive patients and no HIV-negative patients had R-1 resection. Seven HIV-positive patients (7\%) and 2 HIV-negative patients (7\%) developed recurrence. The median disease-free interval was 14 months for HIV-positive patients compared with 10 months for those who were HIV-negative.

Complications of chemotherapy and radiotherapy occurred with similar frequency in the two groups (Table 4). The frequency of afebrile neutropenia was slightly higher in

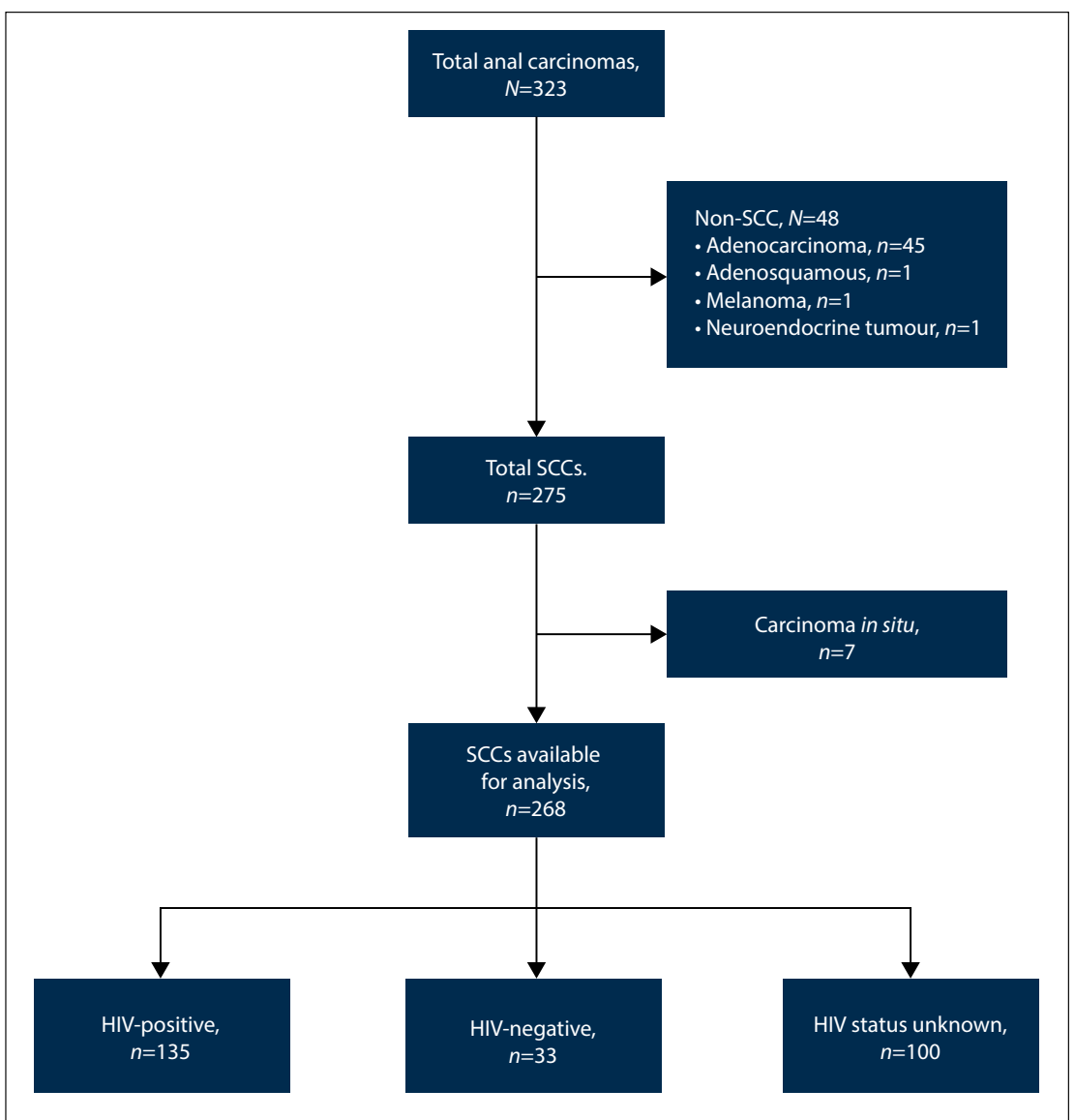

Fig. 1. Flow diagram illustrating the study inclusion and exclusion criteria and describing the patients with anal squamous cell carcinoma eligible for initial analysis. (SCC = squamous cell carcinoma.)
HIV-positive than in HIV-negative patients, and anaemia was only seen in HIV-positive patients.

The median follow-up for the HIVpositive patients was 9 (IQR 3 - 21) months and that for the HIV-negative patients 11.5 (IQR 5 - 24.5) months. Fig. 4 shows the Kaplan-Meier survival curves. Overall, the 5-year survival rate for HIV-positive and negative patients was $57 \%$ and $63 \%$, respectively $(p=0.0240)$. Median survival was 23.1 months for HIV-negative patients and 29.8 months for HIV-positive patients (not statistically significant).

\section{Discussion}

This study presents an audit of anal squamous cell carcinoma and analyses differences in the demographic profile, clinicopathological features, treatment tolerance and final outcome between HIVpositive and HIV-negative patients with squamous cell carcinoma of the anus in our unit. In evaluating the influence of HIV infection on presentation of these patients and outcome of treatment, we have made a number of observations.

The median duration of symptoms in HIVnegative and positive patients was similar. Reasons for delay in treatment included late presentation by the patient in seeking medical attention, incorrect diagnosis by the primary doctor, and delayed referral by the referring doctor.

According to the 2014 SA registry, anal cancer comprised $0.33 \%$ of cancers in females v. $0.26 \%$ in males, with 125 new cases annually in females v. 95 in males. ${ }^{[16]}$ The present study demonstrated an increasing accrual of patients with squamous cell anal carcinoma over the years. The rising rate of anal cancer can partly be explained by the longer life expectancy of HIV-infected patients on ART and an increased incidence of HPV infections, ${ }^{[15,17]}$ in addition to possible

Table 1. Profile of 168 patients with anal squamous cell carcinoma stratified according to HIV status ${ }^{\dagger}$

\begin{tabular}{llll}
\multicolumn{4}{l}{ Table 1. Profile of $\mathbf{1 6 8}$ patients with anal squamous cell carcinoma stratified according to HIV status ${ }^{\dagger}$} \\
\hline Characteristics & Total $(\boldsymbol{N = 2 6 8 )}$ & HIV-positive $(\boldsymbol{n}=\mathbf{1 3 5})$ & HIV-negative $(\boldsymbol{n}=\mathbf{3 3})$ \\
\hline Age (years), median (IQR) & $44(37-57)$ & $40(34-45)^{*}$ & $32(48-58)^{*}$ \\
Male, $n$ & 84 & 98 & 21 \\
Female, $n$ & 184 & $1: 2.7$ & $1: 1.8$ \\
Male/female ratio & $1: 2.2$ & 355 & $\mathrm{n} / \mathrm{a}$ \\
CD4+ count (cells/ $\mu \mathrm{L})$, median & $\mathrm{n} / \mathrm{a}$ & 50 & $\mathrm{n} / \mathrm{a}$ \\
Antiretroviral therapy & $\mathrm{n} / \mathrm{a}$ & 90 & 17 \\
Margin, $n$ & 183 & 45 & 16 \\
Canal, $n$ & 85 & $2: 1$ & $1: 1$ \\
Margin/canal distribution ratio & $2.2: 1$ & & \\
IQR $=$ interquartile range; $\mathrm{n} / \mathrm{a}=$ not applicable. & & &
\end{tabular}


increasing awareness of the disease on the part of patients and doctors and availability of the oncology services at IALCH.

Anal cancer traditionally affects older women more than men, but the incidence is increasing in younger women, homosexual men and patients with HIV infection. ${ }^{[7]}$ This is not surprising, because HPV infection in immunocompromised patients tends to act synergistically with HIV and leads to eventual carcinogenesis. ${ }^{[8]}$ The female predominance that was observed in both groups in this study is in keeping with the SA and international literature, which also reports a female preponderance. ${ }^{[4,9,14,17]}$ Notably, however, the relative preponderance

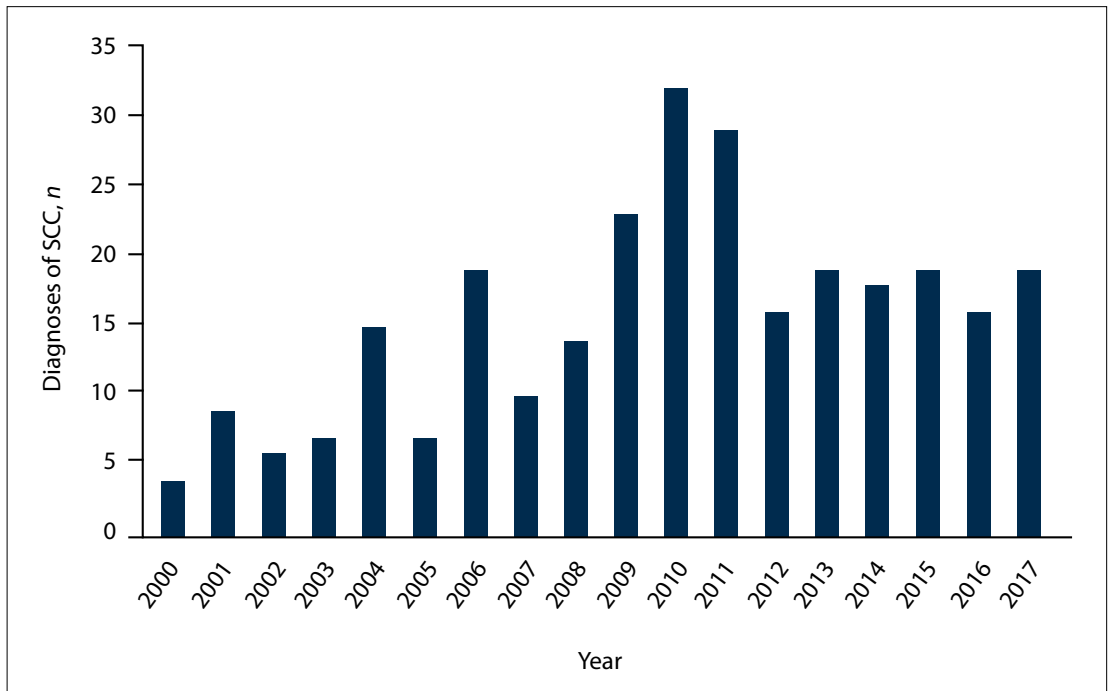

Fig. 2. Annual accrual of patients with SCC $(\mathrm{p}=0.680)$. (SCC = squamous cell carcinoma.)

of female patients was more pronounced in the HIV-positive group. This relative predominance is not surprising, since various surveys show that women continue to account for a disproportionate percentage of new HIV infections among adults in subSaharan Africa, where they comprise $59 \%$ of the affected population. ${ }^{[18,19]}$ One reason for this difference is that men are less likely than women to use health services and are less likely to take an HIV test. ${ }^{[19]}$

The mean age of presentation for the whole group of 48 years was approximately a decade younger than the 56 years reported in the international literature. ${ }^{[1,12,14]}$ In addition, HIV-positive patients presented over a decade earlier than their HIVnegative counterparts. The younger age of presentation in HIV-positive patients confirms our hypothesis that patients with anal squamous cell carcinoma and HIV co-infection present at an earlier age than those without HIV infection. This tendency for HIV-positive patients to present at a younger age has been observed by others. ${ }^{[7,11,13,17]}$

Anal margin cancers were twice as common as anal canal cancers in our study

\begin{tabular}{lll}
\multicolumn{2}{l}{ Table 2. Primary management of patients with anal squamous cell carcinoma } \\
\hline Management & HIV-positive $(\boldsymbol{N}=\mathbf{1 3 5}), \boldsymbol{n}(\%) / \boldsymbol{n}$ & HIV-negative $(\boldsymbol{N}=\mathbf{3 3}), \boldsymbol{n}(\%) / \boldsymbol{n}$ \\
\hline Definitive therapy & $69(51.1)$ & $17(51.5)$ \\
Chemoradiation & 58 & 17 \\
Chemotherapy & 4 & 0 \\
Radiotherapy & 7 & 0 \\
Palliative therapy & $32(23.7)$ & $12(36.4)$ \\
Radiotherapy & 20 & 7 \\
Chemotherapy + radiation & 12 & 3 \\
Chemotherapy & 0 & 2 \\
No treatment & $34(24.4)$ & $4(12.1)$ \\
Did not arrive & 15 & 1 \\
Planned for treatment - did not return & 10 & 2 \\
Poor general condition & 5 & 0 \\
Complete surgical resection & 3 & 1 \\
Previous radiotherapy & 1 & 0
\end{tabular}

Table 3. Surgical resection in patients with anal squamous cell carcinoma

\begin{tabular}{lll}
\hline Surgical management & HIV-positive $(N=\mathbf{1 3 5}), \boldsymbol{n}(\%) / \boldsymbol{n}$ & HIV-negative $(\boldsymbol{N}=\mathbf{3 3}), \boldsymbol{n}(\%) / \boldsymbol{n}$ \\
\hline Resection & $17(12.6)$ & $3(9.0)$ \\
Up-front resection & $8(5.9)$ & $1(3.0)$ \\
Salvage resection & $9(6.7)$ & $2(6.1)$ \\
Refused resection & 1 & 1 \\
Trial of dissection & 1 & 0 \\
Type of surgery & & \\
WLE & 11 & 2 \\
A-P resection & 6 & 1
\end{tabular}

WLE $=$ wide local excision; A-P = abdominoperineal. 
group as a whole. On subanalysis according to HIV status, anal margin cancers were twice as common as anal canal cancers in HIVpositive patients, whereas the disease occurred with similar frequency in the anal canal and the anal margin in HIV-negative patients. It therefore appears that the HIV-positive patients added considerably

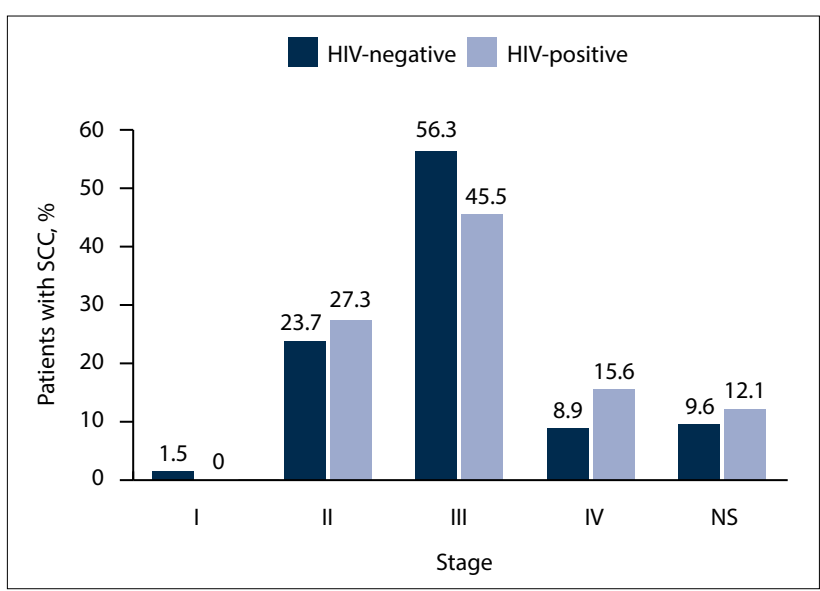

Fig. 3. Staging in patients with anal SCC ( $\mathrm{p}=0.680$, Fisher's exact test.). (SCC = squamous cell carcinoma; $N S=$ not staged.$)$

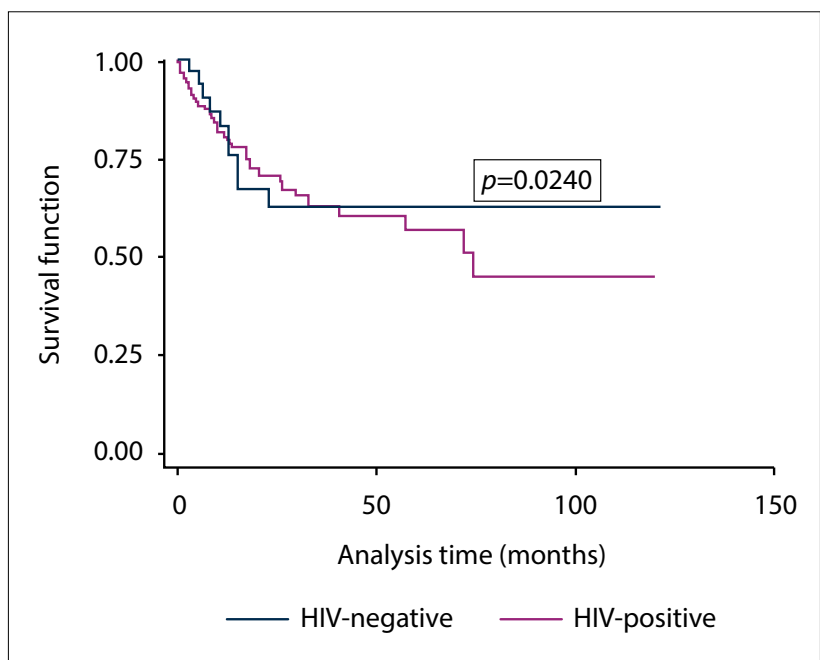

Fig. 4. Survival analysis in HIV-positive and HIV-negative patients with SCC. The difference in overall survival between HIV-positive and negative patients was statistically significant $(\mathrm{p}=0.0240) . \quad(S C C=$ squamous cell carcinoma.) to the number of cancers in the anal margin, which led to the whole group demonstrating an anal margin preponderance. This finding of anal margin preponderance is in contrast to other studies on anal cancer, which show a preponderance of anal canal cancers. ${ }^{[15]}$ Another local study on anal cancer similarly observed a preponderance of anal margin lesions. ${ }^{[20]}$ A limitation of previous international studies on anal cancer is their tendency to consider anal cancer as a homogeneous entity or to describe one or another of the disease sites. ${ }^{[1,20]}$

It was interesting to observe that HIV-negative patients presented with a higher proportion of stage IV disease than HIV-positive patients (16\% v. 9\%) (Fig. 3). These HIV-negative patients were of older age and mostly presented with lung metastases. We believe that this observation cannot be generalised to all patients with squamous cell anal carcinoma, as the numbers in our series were small.

The majority of both HIV-positive and HIV-negative patients presented with locally advanced stage disease owing to late presentation, which resulted in only $13 \%$ and $9 \%$, respectively, meeting the criteria for resection. The fact that only about half of the patients in both groups were eligible for definitive chemoradiation can also be attributed to presentation with locally advanced disease, since only a small proportion had metastatic disease. Inasmuch as immunocompromised patients are thought to have more biologically aggressive tumours, independent of the lesion's actual histological grade, ${ }^{[10]}$ it is counter-intuitive that proportions of patients who were not eligible for definitive therapy were similar in the two groups. However, the small number of HIV-negative patients makes comparison difficult.

Evidence seems to suggest that, in the modern era of ART, anal cancer recurrence and survival outcomes are similar in HIVinfected and HIV-uninfected patients, but HIV-infected patients may experience more toxicity ${ }^{[15]}$ because of altered sensitivity to radiotherapy. ${ }^{[21]}$ However, the present study showed tolerance to chemotherapy and radiotherapy to be similar in the two groups. Anaemia was observed only in HIV-positive patients, but again the number of HIV-negative patients was too small to make a statistical comparison. Studies with larger numbers will assist in determining whether or not toxicity is increased in HIV-positive patients. Fiveyear survival was significantly longer for HIV-negative patients than for those who were HIV-positive, supporting the view that HIV-positive patients with anal cancer tend to do badly in terms of survival. According to the SEER data, the 5-year survival rate in the USA is $68.2 \% .{ }^{[2]}$ An SA study by Robertson et al., ${ }^{[2]}$ describing only anal canal squamous cell carcinoma, reported a 5-year survival rate of $65.6 \%$, which is slightly higher than the $57 \%$ and $63 \%$ for HIVpositive and negative patients, respectively, in the present series.

Table 4. Complications of treatment in 129 patients with anal squamous cell carcinoma receiving oncological therapy

\begin{tabular}{|c|c|c|}
\hline Complications & HIV-positive $(N=99), n(\%) / n$ & HIV-negative $(N=30), n(\%) / n$ \\
\hline Total with complications & $33(33.3)$ & $10(33.3)$ \\
\hline Total with chemotherapy complications & $20(20.2)^{\star}$ & $4(13.3)$ \\
\hline Afebrile neutropenia & $10(10.1)$ & $2(6.7)$ \\
\hline Anaemia & 7 & 0 \\
\hline Miscellaneous & 4 & 2 \\
\hline Total with radiation complications & $21(21.2)$ & $6(20.0)$ \\
\hline Skin reaction & 16 & 6 \\
\hline Radiation proctitis & 3 & 0 \\
\hline Radiation cystitis & 1 & 0 \\
\hline Rectovaginal fistula & 1 & 0 \\
\hline
\end{tabular}




\section{Study limitations}

This study has certain limitations. The number of HIV-negative patients was very small. We were not able to estimate the duration of HIV infection between seroconversion and the development of anal cancer, or the duration of immune reconstitution after commencement of antiretroviral treatment. Knowledge of these two variables could contribute to understanding of the role of HIV infection and its treatment on carcinogenesis. We also did not evaluate individual patients' sexual practices owing to the conservative cultural traditions in this community. Despite these limitations, the study accrued large enough numbers to reach important conclusions.

\section{Conclusions}

Although anal squamous cell carcinoma is a rare malignancy, in this series the prevalence was higher in HIV-positive patients than in those who were HIV-negative. HIV-positive patients were younger than their HIV-negative counterparts. The side-effects of chemoradiation occurred with similar frequency in the two groups, although anaemia was seen only in HIV-positive patients. Median survival of HIV-positive patients was lower. We recommend that patients with precursor lesions such as AIN and HIV co-infection should be offered closer surveillance, enabling earlier detection and appropriate treatment of invasive lesions. We are in agreement with others that condylomas should not be cauterised, but should always be resected and sent for histological evaluation. ${ }^{[20]}$ Since HPV is a risk factor and a causative factor in $90 \%$ of anal cancers, ${ }^{[15]}$ we agree with other authors that HPV vaccination should be considered as an intervention for the prevention of anal cancer. ${ }^{[9,23,24]}$

Declaration. Publication was a requirement for NPZ's MMed (Radiation Oncology) degree.

Acknowledgements. The authors thank Prof. B Sartorius, University of KwaZulu-Natal, for statistical analysis.

Author contributions. Study design: TEM, NPZ. Data collection: NPZ. Data analysis: NPZ, SN, TEM. Draft version: NPZ, SN, TEM. Final version: NPZ, SN, TEM.

Funding. None.

\section{Conflicts of interest. None.}

Statement. Presented at the 46th Meeting of the Surgical Research Society of Southern Africa in Mthatha, Eastern Cape Province, South Africa, 21 22 June 2018.
1. Siegel RL, Miller KD, Jemal A. Cancer statistics. CA Cancer J Clin 2019;69(1):7-34. https://doi. org/10.3322/caac. 21551

2. National Cancer Institute. Surveillance, Epidemiology, and End Results Program. Cancer stat facts: 2. National Cancer Institute. Surveillance, Epidemiology, and End Results Program. Cancer stat
Anal cancer. 2019. https://seer.cancer.gov/statfacts/html/anus.html (accessed 6 June 2019).

3. Chiao E, Krown S, Stier E, Schrag D. A population-based analysis of temporal trends in the incidence of squamous anal canal cancer in relation to the HIV epidemic. J Acquir Immune Defic Syndr 2005;40(4):451-455. https://doi.org/10.1097/01.qai.0000159669.80207.12

4. Johnson L, Madeleine M, Newcomer L, Schwartz S, Daling J. Anal cancer incidence and survival: The surveillance, epidemiology, and end results experience, 1973 - 2000. Cancer 2004;101(2):281288. https://doi.org/10.1002/cncr.20364

5. Palefsky JM. Anal human papillomavirus infection and anal cancer in HIV-positive individuals: An emerging problem. AIDS 1994;8(3):283-296.

6. Kan M, Wong PHP, Press N, Wiseman SM. Colorectal and anal cancer in HIV/AIDS patients: A comprehensive review. Expert Rev Anticancer Ther 2014;14(4):395-405. https://doi.org/10.158 6/14737140.2013.877843

7. Kim J, Sarani B, Orkin B, et al. HIV-positive patients with anal carcinoma have poorer treatment tolerance and outcome than HIV-negative patients. Dis Colon Rectum 2001;44(10):1496-1502. https://doi.org/10.1007/BF02234605

8. Palefsky JM, Holly EA. Chapter 6: Immunosuppression and co-infection with HIV. J Natl Cancer Inst Monogr 2003;2003(31):41-46. https://doi.org/10.1093/oxfordjournals.jncimonographs.a003481 Colón-López V, Shiels MS, Machin M, et al. Anal cancer risk among people with HIV infection in the United States. J Clin Oncol 2018;36(1):68-75. https://doi.org/10.1200/JCO.2017.74.9291

10. Hoots BE, Palefsky JM, Pimenta JM, Smith JS. Human papillomavirus type distribution in anal cancer and anal intraepithelial lesions. Int J Cancer 2009;124(10):2375-2383. https://doi. org/10.1002/ijc.24215

11. Crum-Cianflone NF, Hullsiek KH, Marconi VC, et al. Anal cancers among HIV-infected persons: HAART is not slowing rising incidence. AIDS 2010;24(4):535-543. https://doi.org/10.1097/ QAD.0b013e328331f6e2

12. Hurley J, Franco S, Gomez-Fernandez C, et al. Breast cancer and human immunodeficiency virus: A report of 20 cases. Clin Breast Cancer 2001;2(3):215-220. https://doi.org/10.3816/CBC.2001.n.024

13. Wang C-CJ, Palefsky JM. HPV-associated anal cancer in the HIV/AIDS patient. In: Meyers C, ed. HIV/AIDS-Associated Viral Oncogenesis: Cancer Treatment Research. Cham, Switzerland: ed. Hiv/AIDS-Associated Viral Oncogenesis: Cancer Treatment Reser
Springer, 2018:183-209. https://doi.org/10.1007/978-3-030-03502-0_7

14. Rousseau DL jr, Thomas CR jr, Petrelli NJ, Kahlenberg MS. Squamous cell carcinoma of the anal 4. Rousseau DL jr, Thomas CR jr, Petrelli NJ, Kahlenberg MS. Squamous cell carcin
canal. Surg Oncol 2005;14(3):121-132. https://doi.org/10.1016/j.suronc.2005.07.002

15. Duncan KC, Chan KJ, Chiu CG, et al. HAART slows progression to anal cancer in HIV-infected MSM. AIDS 2015;29(3):305-311. https://doi.org/10.1097/QAD.0000000000000537

16. Summary statistics of cancer diagnosed histologically in 2014. http://www.nicd.ac.za/wp-content/ uploads/2017/03/2014-NCR-tables-1.pdf (accessed 22 February 2017).

17. Vatra B, Sobhani I, Aparicio T, et al. Anal canal squamous-cell carcinomas in HIV-positive patients: Clinical features, treatments and prognosis. Gastroenterol Clin Biol 2002;26(2):150-156.

18. Joint United Nations Programme on HIV and AIDS (UNAIDS). UNAIDS data 2018. State of the Epidemic. https://www.unaids.org/sites/default/files/media_asset/unaids-data-2018_en.pdf (accessed 24 August 2019).

19. Joint United Nations Programme on HIV and AIDS (UNAIDS). UNAIDS 2017: A snapshot of men and HIV in South Africa. https://webcache.googleusercontent.com/search?q=cache:RLeJH7TTbzs J:https://www.unaids.org/en/file/111992/download\%3Ftoken\%3DHCocZ_Hh+\&cd=1\&hl=en\&ct= f:https://www. unaids.org/en/file/111992/download\%
clnk\&gl=za\&client=safari (accessed 24 August 2019).

20. Ntombela X, Sartorius B, Madiba T, Govender P. The clinicopathologic spectrum of anal cancer in KwaZulu-Natal Province, South Africa: Analysis of a provincial database. Cancer Epidemiol in KwaZulu-Natal Province, South Africa: Analysis of a provin

21. Bjørge T, Engeland A, Luostarinen T, et al. Human papillomavirus infection as a risk factor for anal and perianal skin cancer in a prospective study. Br J Cancer 2002;87(1):61-64. https://doi. org/10.1038/sj.bjc. 6600350

22. Robertson B, Shepherd L, Abratt RP, Hunter A, Goldberg P. Treatment of carcinoma of the anal canal at Groote Schuur Hospital. S Afr Med J 2012;102(6):558-561.

23. Medford RJ, Salit IE. Anal cancer and intraepithelial neoplasia: Epidemiology, screening and prevention of a sexually transmitted disease. Can Med Assoc J 2015;187(2):111-115. https://doi. org $/ 10.1503 / \mathrm{cmaj} .140476$

24. Palefsky JM, Giuliano AR, Goldstone S, et al. HPV vaccine against anal HPV infection and anal intraepithelial neoplasia. N Engl J Med 2011;365(17):1576-1585. https://doi.org/10.1056/ NEJMoa1010971

Accepted 4 September 2019. 